\title{
Improved system blind identification based on second-order cyclostationary statistics: A group delay approach
}

\author{
P V S GIRIDHAR $^{1}$ and S V NARASIMHAN ${ }^{2}$ \\ ${ }^{1}$ Dept. of Electronics \& Communication Engineering, Karnataka Regional \\ Engineering College, Surathkal 574 157, India \\ Present address: Centre for Development of Telematics, Bangalore 560 052, \\ India \\ ${ }^{2}$ Aerospace Electronics Division, National Aerospace Laboratories, Bangalore \\ 560017 , India \\ e-mail:svn@css.cmmacs.ernet.in
}

\begin{abstract}
An improved system blind identification method based on secondorder cyclostationary statistics and the properties of group delay, has been proposed. This is achieved by applying a correction to the estimated phase (by the spectral correlation density of the system output) for the poles, in the group delay domain. The results indicate a significant improvement in system blind identification, in terms of root mean square error. Depending upon the signalto-noise ratio, the improvement in percentage normalized mean square error ranges between 20 and $50 \%$.
\end{abstract}

Keywords. Cyclostationarity; system blind identification; group delay functions.

\section{Introduction}

The system blind identification is an important engineering problem, in many fields. For minimum phase systems, identification using autocorrelation, based on stationarity assumption, is valid. Autocorrelation based methods are limited to minimum phase systems, as they cannot distinguish between minimum and maximum phase system roots. This is due to the fact that an autocorrelation function has complete information about only the spectral magnitude and not the phase. However, many systems, like data communication channels, acoustic paths and vocal tract, are of non-minimum phase nature and their actual identification is essential. Higher order statistics (HOS), like the bispectrum, have complete system phase information in a hidden manner and many methods to extract phase from them have been proposed (Giannakis \& Mendel 1988; Nikias 1988; Narasimhan et al 1992). However, these methods involve large amounts of data and processing, and in some cases minimization of a nonlinear cost function (Hatzinakos 1994). Further, the bispectrum, the easiest to compute among the higher order statistics, is valid only when the signals involved have non-symmetric amplitude distributions and hence is not applicable to signals which are Gaussian. 
Systems or signals whose spectral characteristics change periodically with time, are said to be cyclostationary in nature and such signals exhibit spectral correlation between frequency components (Gardner 1991). Many man-made signals in the field of communication and rotating machinery, and many natural signals like astronomical and weather, possess cyclostationarity. However, for a stationary signal/system, this cyclic correlation is zero. Even with signals which are not cyclostationary, in certain cases, it is possible to take advantage of this property by artificially inducing it.

In the last decade, there has been considerable research on achieving blind identification of a system, based on cyclostationarity (Hatzinakos 1994; Li \& Ding 1994; Tsatsanis 1997). In contrast to HOS based methods, the advantages of methods based on secondorder cyclic statistics are: (1) fewer data samples are required, and less complexity is encountered in the estimation of the cyclic second-order statistics, (2) there are no restrictions on the distribution of the input data, and (3) they are insensitive to stationary additive noise. Blind identification has been achieved by fractionally sampling the channel output and computing the complex cepstrum of the output cyclic autocorrelation (Hatzinakos 1994). The computation of complex cepstrum poses problems when the zeros are very close to unit circle. By introducing cyclostationarity at the transmitter rather than at the receiver and using a subspace approach, good blind channel equalization has been achieved in the absence of observation noise, without any constraints on the location of channel zeros (Tsatsani1997).

Blind identification of ARMA systems based on second-order cyclic statistics both by the parametric and nonparametric methods is investigated ( $\mathrm{Li} \& \mathrm{Ding}$ 1994). The nonparametric method directly identifies the system phase from the spectral correlation density (SCD) of the channel output. On the other hand, the parametric method directly identifies the poles and zeros of the mixed phase ARMA channels using SCD of the channel output. In the nonparametric approach (Hatzinakos 1994; Li \& Ding 1994), at frequencies $2 \pi / p, p$ being the oversampling rate, the phase information either cannot be computed or is in error. The parametric method requires advance information about the order of the system. Also to reduce the phase estimation errors at the poles, a hybrid method (Li \& Ding 1994), in which the poles of the channel are predetermined using the parametric method, has been proposed. Compared to the nonparametric methods, generally, the performance of a parametric method is limited to the high signal-to-noise ratio condition and is applicable only when the assumed model matches the process under consideration.

Group delay (GD), the negative derivative of phase of the Fourier transform, is known for its high resolution and ease of processing (Yegnanarayana et al 1984). The high resolution is due to the fast transitions in phase (compared to that of the spectral magnitude) leading to better localization of the GD. The ease of processing is due to the additive nature of phase which transforms convolution in time to addition in GD domain. Further, this property enables decomposition of a signal to the minimum and maximum phase components (Yegnanarayana 1981), and pole-zero components, by simple addition and/or subtraction. These properties of GD have been exploited for system identification using bispectrum (Narasimha et al 1992b).

In this paper, it is proposed to use the GD approach for the improved blind identification based on cyclostationarity. This is achieved by applying a correction to the estimated phase (by the spectral correlation density of the system output (Li \& Ding 1994) for the poles, in the GD domain. The GD domain not only facilitates easy phase correction but also, being a nonparametric method, provides better performance at low signal-to-noise ratios and is 


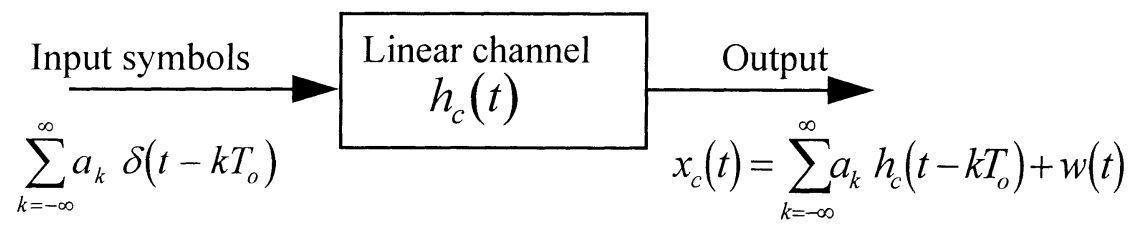

Figure 1. Baseband representation of a PAM data communication system.

applicable to signals in general. It has been found that there is a significant improvement in the blind identification of a system, in terms of root mean square error.

\section{Blind identification based on cyclostationarity}

\subsection{Cyclostationarity of oversampled channel output (Li \& Ding 1994)}

For the identification method, a baseband representation of the pulse amplitude modulated (PAM) communication system shown in figure 1 is considered. $h_{c}(t)$ is the composite channel impulse response that includes all interconnections between the symbol generator and the output of the receiving filter. Also, the channel is assumed to be linear, timeinvariant, causal and bounded input-bounded output stable. The symbol source generates a zero mean i.i.d. (independent, identically distributed) sequence $\left\{a_{k}\right\}$, each element of which comes from a constellation of PAM symbols. For this, the continuous time received signal is given by

$$
x_{c}(t)=\sum_{l} a(l) h_{c}\left(t-l T_{0}-d\right)+w(t)
$$

where $T_{0}^{-1}$ is the baud rate (the rate at which signal level is changed, depends upon the nature of the format used to represent the digital data), $d \in\left[0, T_{0}\right.$ (i.e., $0 \leq d<T_{0}$ ) is the unknown propagation delay and $w(t)$ is the additive noise at the receiver. If $T_{s}$ is the sampling period and defining

$$
h(n)=\left.h_{c}(t-d)\right|_{t=n T_{s}}, \quad w(n)=\left.w(t)\right|_{t=n T_{s}},
$$

the discrete-time model is given by

$$
x(n)=\left.x_{c}(t)\right|_{t=n T_{s}}=\sum_{l} a(l) h(n-l p)+w(n), \quad \text { where } p=T_{0} / T_{S},
$$

and where $p \in Z$ (set of positive integers) and $p \neq 1$, is the rate at which the channel output is oversampled w.r.t. the baud rate. This results in a discrete cyclostationary process. The correlation function of $x(n)$ is defined as

$$
R_{x}(n+m, n)=E\left\{x(n+m) x^{*}(n)\right\} .
$$

Substituting for $x(n)$ and $x(n+m)$ in (3) and assuming that $a(n)$ and $w(n)$ are independent,

$$
R_{x}(n+m, n)=E\left\{\left[\sum_{r} a(r) h(n+m-r p) \sum_{k} a^{*}(k) h^{*}(n-k p)\right]\right\}+N_{0} \delta(m),
$$


$x(n)$ is cyclostationary with period $p$ since $R_{x}(n+m+p, n+p)=R_{x}(n+m, n), n, m \in Z$. The cyclic correlation function of the discrete process $x(n)$ is defined as

$$
R_{x}^{(l \beta)}(m)=\sum_{n=0}^{p-1} R_{x}(n+m, n) e^{-j n l \beta}, \beta=2 \pi / p, \quad \text { for } l \in Z .
$$

Substituting (4) in (5),

$$
R_{x}^{(l \beta)}(m)=\sum_{n=0}^{p-1} E\left\{\left[\sum_{r} a(r) h(n+m-r p) \sum_{k} a^{*}(k) h^{*}(n-k p)\right]\right\}+N_{0} \delta(m) .
$$

The cyclic spectrum or the spectral correlation density (SCD) is obtained by,

$$
S_{x}^{(l \beta)}(\omega)=\sum_{m=-\infty}^{\infty} R_{x}^{l \beta}(m) e^{-j m \omega} .
$$

Substituting (6) in (7)

$$
S_{x}^{(l \beta)}(\omega)=E\left\{\left|a_{n}\right|^{2}\right\} H\left(e^{j \omega}\right) \sum_{n} \sum_{k}\left[h^{*}(n-k p) e^{j(n-k p)(\omega-l \beta)} e^{j k p l \beta}\right]+p N_{0} \delta(l) .
$$

If $n-k p=u$, since $\beta=2 \pi / p, e^{j k p l \beta}=1$ for $k, l \in Z$,

$$
\begin{aligned}
& S_{x}^{l \beta}(\omega)=E\left\{\left|a_{n}\right|^{2}\right\} H\left(e^{j \omega}\right) \sum_{u} h^{*}(u) e^{j u(\omega-l \beta)}+p N_{0} \delta(l), \\
& S_{x}^{l \beta}(\omega)=E\left\{\left|a_{n}\right|^{2}\right\} H\left(e^{j \omega}\right) H^{*}\left(e^{j(\omega-l \beta)}\right)+p N_{0} \delta(l) .
\end{aligned}
$$

This is the key relation for channel identification. Further, when $l \neq 0$, the SCD provides noise rejection.

\subsection{Channel identifiability based on output SCD (Li \& Ding 1994)}

The objective is to identify the channel frequency response $H(\omega)$ from the SCD of the channel output $x(n)$ for a zero-mean and i.i.d. input.

2.2a Magnitude estimation: When the linear system output is not corrupted by stationary additive noise, the magnitude can be identified from (8) by setting $l=0$,

$$
\begin{aligned}
& S_{x}^{l \beta}(\omega)=E\left\{\left|a_{n}\right|^{2}\right\} H\left(e^{j \omega}\right) H^{*}\left(e^{j(\omega-l \beta)}\right), \\
& S_{x}^{0}(\omega)=\sigma^{2} H(\omega) H^{*}(\omega), \quad \text { where } \sigma^{2}=E\left\{a_{n}^{2}\right\}, \\
& |H(\omega)|=(1 / \sigma)\left(S_{x}^{0}(\omega)\right)^{1 / 2} .
\end{aligned}
$$

When a strong stationary additive noise is present, an approach by setting $l=1$ in (8) is taken,

$$
\begin{aligned}
& S_{x}^{l \beta}(\omega)=E\left\{|a(n)|^{2}\right\} H(\omega) H^{*}(\omega-l \beta) \quad \beta=2 \pi / p, \quad l= \pm 1, \pm 2, \ldots \\
& S_{x}^{\beta}(\omega)=\sigma^{2} H(\omega) H^{*}(\omega-\beta) .
\end{aligned}
$$


Taking the real part of the logarithm on both sides,

$$
\ln \left(\left|S_{x}^{l}(\omega \mid)=\ln \right| \sigma^{2}|+\ln | H(\omega)|+\ln | H^{*}(\omega-2 \pi / p) \mid .\right.
$$

When $\ln \left(\left|s_{x}^{1}(\omega)\right|\right)$ is finite and none of the zeros of the channel are on the unit circle, then the real cepstra for $H(\omega)$ and $S_{x}^{1}(\omega)$ are defined as,

$$
c_{h}(n)=\operatorname{IDFT}(\ln |H(\omega)|) \quad \text { and } \quad c_{1}(n)=\operatorname{IDFT}\left(\ln \left|S_{x}^{1}(\omega)\right|\right),
$$

where IDFT stands for Inverse Discrete Fourier Transform. If both the cepstra exist, then based on (10), $c_{h}(n)$ and $c_{1}(n)$ are related by,

$$
c_{1}(n)=\ln \left|\sigma^{2}\right| \delta(n)+c_{h}(n)\left(1+e^{j 2 \pi n / p}\right) .
$$

The amplitude of the system transfer function is given by

$$
c_{h}(n)=\left[c_{1}(n)-\delta(n) \ln \left|\sigma^{2}\right|\right] /\left(1+e^{j 2 n \pi / p}\right)
$$

and

$$
|H(\omega)|=\exp \left(\operatorname{DFT}\left(c_{h}(n)\right)\right) .
$$

However, there is no solution available whenever

$$
2 n / p=2 k+1, \quad \text { or } \quad n=\left(k+\frac{1}{2}\right) p, \quad k \in Z .
$$

Thus, as long as $p$ is an odd integer, the amplitude of $H(\omega)$ can be completely identified from the SCD, even when strong stationary noise is present. The above discussed approach is advantageous only when a strong noise is present and a relatively large number of data samples are available.

$2.2 b$ Phase estimation: The phase can be identified from (8) with $l \neq 0$.

$$
S_{x}^{l \beta}(\omega)=E\left\{|a(n)|^{2}\right\} H(\omega) H^{*}(\omega-l \beta), \quad \beta=2 \pi / p, \quad l= \pm 1, \pm 2, \ldots
$$

If $\psi_{l}(\omega)$ and $\Phi(\omega)$ are the phase responses of the $\operatorname{SCD} S_{x}^{l \beta}(\omega)$ and $H(\omega)$ respectively, then according to (12) for $l \neq 0$,

$$
\begin{aligned}
& \Psi_{l}(\omega)=\Phi(\omega)-\Phi(\omega-l \beta), \\
& \Psi_{l}(\omega)=\Phi(\omega)-\Phi(\omega+(2 \pi / p) l), \quad l= \pm 1, \pm 2, \ldots,
\end{aligned}
$$

where $\Psi_{l}(\omega)$ is the unwrapped phase. For an arbitrary channel, the above equations indexed by $l$ are the only sources of phase information. $\Phi(\omega)$ can be obtained from $\Psi_{l}(\omega)$ as given by

$$
\phi(\tau)=\psi_{l}(\tau) /\left(1-e^{-j 2 \pi l \tau / p}\right)
$$

where the cepstra, $\psi_{l}(\tau)$ and $\phi(\tau)$ are the inverse Fourier transforms of $\Psi_{l}(\omega)$ and $\Phi(\omega)$, respectively. No cepstral phase information can be extracted when

$$
1-e^{-j 2 \pi l \tau / p}=0, \quad \text { or } \quad \tau=m p / l, \quad m \in Z .
$$

Also from (14), no additional phase information can be extracted from the SCD by setting $l= \pm 2, \pm 3, \ldots$ and hence the SCD evaluated at $l=1$ is only used. Thus, the phase 
obtained from the incomplete set of cepstral coefficients will be highly inaccurate and hence needs some correction. Therefore, an arbitrary channel transfer function cannot be completely identified from the cyclostationary statistics alone.

\section{Improved system identification by group delay}

As pointed out in the last section, the phase estimated is erroneous due to the incomplete set of cepstral coefficients used. This calls for a phase correction. The phase correction can be made with regard to poles and zeros, involved. For poles, the phase correction has been envisaged by identifying the poles using parametric methods (Li \& Ding 1994). However, this phase information about pole will be erroneous as the parametric methods, severely get affected when the additive noise is strong. Presently, it has been proposed to apply the phase correction in the group delay domain, due to its simplicity and also being a nonparametric approach is less sensitive to the additive noise.

\subsection{Group delay function (Yegnanarayana et al 1984)}

The group delay, $\tau(\omega)$ of a signal with spectrum $X(\omega)=|X(\omega)| e^{j \Phi(\omega)}$ is defined to be the negative derivative of the phase $\Phi(\omega)$.

$$
\tau(\omega)=-\mathrm{d} \Phi(\omega) / \mathrm{d} \omega .
$$

Two group delay functions are defined for a signal, one obtained from the spectral magnitude information, called Magnitude Group Delay (MGD), and the other from the phase of the spectrum, called Phase Group Delay (PGD).

For a mixed phase signal (system), the magnitude and phase of $X(\omega)$ can be expressed as,

$$
\ln |X(\omega)|=\sum_{k=0}^{\infty} u(k) \cos (\omega k)
$$

and

$$
\Phi(\omega)=-\sum_{k=1}^{\infty} v(k) \sin (\omega k),
$$

where $u(k)$ and $v(\mathrm{k})$ are cepstral coefficient sequences of the minimum phase equivalent systems derived from the spectral magnitude and phase respectively. The PGD $\tau_{p}(\omega)$ is given by,

$$
\tau_{p}(\omega)=-\frac{\mathrm{d} \Phi(\omega)}{\mathrm{d} \omega}=\sum_{k=1}^{\infty} k v(k) \cos (\omega k),
$$

and the MGD $\tau_{m}(\omega)$ is given by

$$
\tau_{m}(\omega)=\sum_{k=1}^{\infty} k u(k) \cos (\omega k)
$$

In the case of a minimum phase system, $u(k)=v(k)$. Hence, the MGD and PGD of a minimum phase system are also identical. For a maximum phase system, $u(k)=-v(k)$, 
hence $\tau_{p}(\omega)=-\tau_{m}(\omega)$. However for a mixed phase system, the magnitude and phase are not related by the same set of cepstral coefficients. The phase derived from magnitude corresponds to the phase of a minimum phase equivalent of the signal or system. The PGD of a general system, can be directly obtained from (17) and (18), or from the spectrum as given below,

$$
\tau_{p}(\omega)=\frac{X_{\mathrm{R}}(\omega) Y_{\mathrm{R}}(\omega)+X_{\mathrm{I}}(\omega) Y_{\mathrm{I}}(\omega)}{|X(\omega)|^{2}},
$$

where $\mathrm{R}$ and I refer to the real and imaginary parts. $Y(\omega)$ is the Fourier transform of $y(n)=n x(n)$. If the phase $\Phi(\omega)$ is known, the PGD can be obtained by the allpass sequence $x(n)$ which is the inverse Fourier transform of $e^{j \Phi(\omega)}$.

\subsection{Pole-zero decomposition (Yegnanarayana 1981)}

The decomposition of a signal spectrum into a pole and a zero spectrum is based on the properties of the GD of the minimum phase signal, the MGD (Yegnanarayana 1981). It has been shown that the significant values of MGD for a real pole are close to the origin and, for a second order pole are around the resonance frequency. The MGD for the zeros are the same as those of poles except for the difference in sign. The MGD for a real and a complex pole is positive, while for real and complex zero is negative.

The decomposition of the pole and zero components of the spectrum is obtained by separating the positive and negative parts of the MGD. If $\left[\tau_{m}(\omega)\right]^{+}$and $\left[\tau_{m}(\omega)\right]^{-}$represent the positive and negative parts of the MGD respectively, then the cepstral coefficients for the pole part, $u^{+}(n)$ and for the zero part, $u^{-}(n)$ are related to $\left[\tau_{m}(\omega)\right]^{+}$and $\left[\tau_{m}(\omega)\right]^{-}$ respectively by,

$$
\begin{aligned}
& {\left[\tau_{m}(\omega)\right]^{+}=C+\sum_{k=1}^{\infty} k u^{+}(k) \cos (\omega k),} \\
& {\left[\tau_{m}(\omega)\right]^{-}=C+\sum_{k=1}^{\infty} k u^{-}(k) \cos (\omega k),}
\end{aligned}
$$

where $C$ is the average value and does not contribute to the shape of the spectrum. The first few cepstral coefficients determine the log spectrum as they are its Fourier coefficients. Hence, cepstrally smooth spectra for poles and zeros separately can be obtained by considering only the first few cepstral coefficients in $u^{+}(n)$ and $u^{-}(n)$. These properties enable the pole-zero decomposition.

\subsection{Phase correction}

The errors in estimating the phase by the nonparametric approach, explained in $\S 2$, affect the location of both poles and zeros. For a physically realizable system, all the poles must be of minimum phase, i.e. all poles must be inside the unit circle. From the pole zero decomposition property of the MGD, it is possible to separate poles and zeros in the group delay domain. The poles correspond to the positive portion and the zeros to the negative portion, of the MGD, (21) and (22).

In the group delay domain, the MGD contains complete information about poles. Correction can be achieved in the group delay domain. In the PGD, the correction for the 
poles is done by identifying the positive parts of the MGD and shifting these values to the corresponding regions in the PGD. Further, such correction will result in some discontinuity in the modified PGD. These discontinuities can be smoothed by considering only first $M$ Fourier coefficients of the PGD and the modified smoothed PGD is given by,

$$
\tilde{\tau}_{p}(\omega)=\sum_{k=1}^{M} k c(k) \cos (\omega k),
$$

where $c(k)$ are the cepstral coefficients derived from the modified PGD. Then, the system phase is given by

$$
\tilde{\Phi}(\omega)=-\sum_{k=1}^{M} c(k) \sin (\omega k) .
$$

This corrected phase can be used for the channel identification along with the estimated magnitude.

However, because of the ambiguity about zeros in MGD, as to whether they are minimum or maximum phase, the same correction cannot be applied to the zeros.

\subsection{Algorithm for improved system/channel blind identification based on GD}

(1) Generate the PAM data output: $x(n)=\sum_{l} a(l) h(n-l p)+w(n)$, where $p$ is the oversampling rate. The input sequence $\{a(n)\}$ is an i.i.d. four-level PAM signal. $w(n)$ is a stationary white Gaussian noise. Oversampling the input sequence $\{a(n)\}$ gives the equivalent oversampled channel output. The oversampled input is obtained by just inserting $(p-1)$ zeros between two symbols.

(2) Estimate the SCD of the received sequence: The spectral correlation density function is estimated by spectrally smoothing the spectral correlation products $X(f-\alpha / 2)$ $X^{*}(f+\alpha / 2)$.

(3) Estimate the spectral magnitude and phase of channel transfer function: The magnitude is identified by (9) or (11) depending on whether noise is considered or not. The phase is identified using (14).

(4) Correction of the phase estimate by group delay approach: The MGD of the estimated channel is determined using (17a) and (19), while PGD is determined from the estimated phase from the SCD, as mentioned in $\S 3.1$ using (20). The phase correction is then performed as explained in $\S 3.3$ and the corrected phase is determined as per (23).

(5) Identifying the system / channel: The estimated frequency response of the channel is obtained by combining the magnitude and phase information calculated in the previous step. $\tilde{H}(\omega)=|\tilde{H}(\omega)| e^{j \tilde{\Phi}(\omega)}$, where $|\tilde{H}(\omega)|$ is the estimated magnitude and $\tilde{\Phi}(\omega)$ is the corrected phase estimate. The estimate of the channel impulse response is determined by taking the inverse Fourier transform of $\tilde{H}(\omega)$.

\section{Simulation results}

The two-system functions considered for blind channel identification are:

$$
H_{1}(z)=\frac{1+1.50000 z^{-1}}{1-1.64453 z^{-1}+0.740818 z^{-2}}
$$


and

$$
H_{2}(z)=\frac{1-0.6000 z^{-1}-0.3937 z^{-3}}{1-0.6561 z^{-4}},
$$

$H_{1}(z)$ has poles at $(0.82227 \pm 0.25436 j)$ and a zero at $-1.5 . H_{2}(z)$ has pole at $( \pm 0.6364 \pm 0.6364 j)$ and zeros at $(-0.5890 \pm 0.5780 j)$ and 0.5781 .

The mean estimate $\hat{h}(n)$ and the root mean square error (RMSE) $\sigma(n)$, used as performance indices are given by

$$
\hat{h}(n)=(1 / 50) \sum_{i=1}^{50} \hat{h}_{i}(n) \text { and } \sigma(n)=\left\{(1 / 50) \sum_{i=1}^{50}\left[h(n)-\hat{h}_{i}(n)\right]^{2}\right\}^{1 / 2} .
$$

Further, to quantify the performance, the percentage normalized mean square error (Li \& Ding 1994), \%NMSE $=100 \times E\left[\sum_{n}\left\{\hat{h}_{i}(n)-h(n)\right\}^{2} / \sum_{n} h^{2}(n)\right]$ is also used. Here, $\hat{h}_{i}(n)$ is the estimated impulse response for the $i$ th trial and $h(n)$ is the true impulse response.

The simulations are carried over on these two channels, under noiseless condition, with an oversampling rate of $p=4$ and a data length of $N=4,096$. The input i.i.d. sequence $\{a(n)\}$ used is a four level PAM signal with symbols $\{-3,-1,1,3\}$.

The SCD is estimated by spectrally smoothing the symmetrical correlation products $X(f-\alpha / 2) X^{*}(f+\alpha / 2)$. The spectral smoothing is done by boxcar over 31 products,
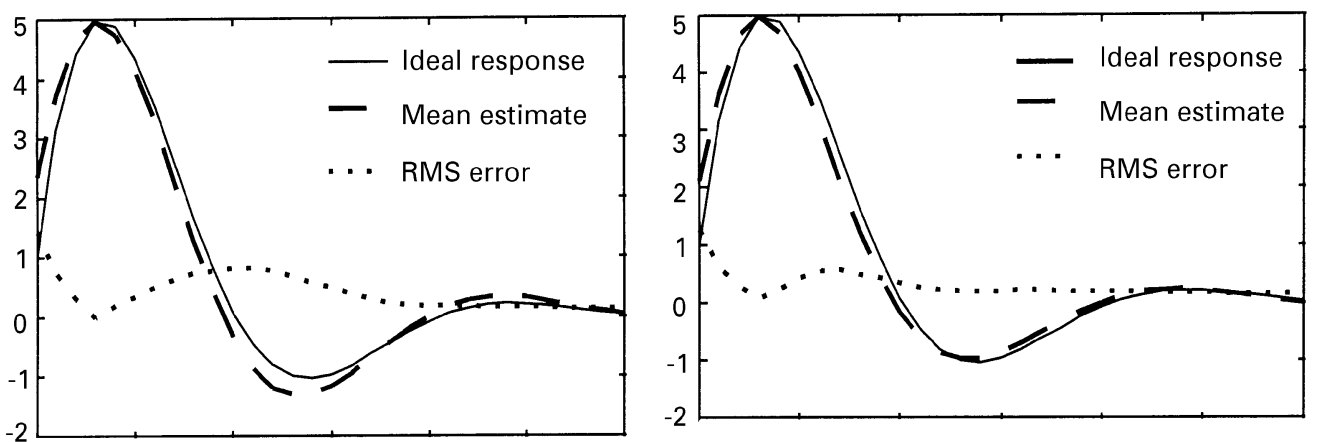

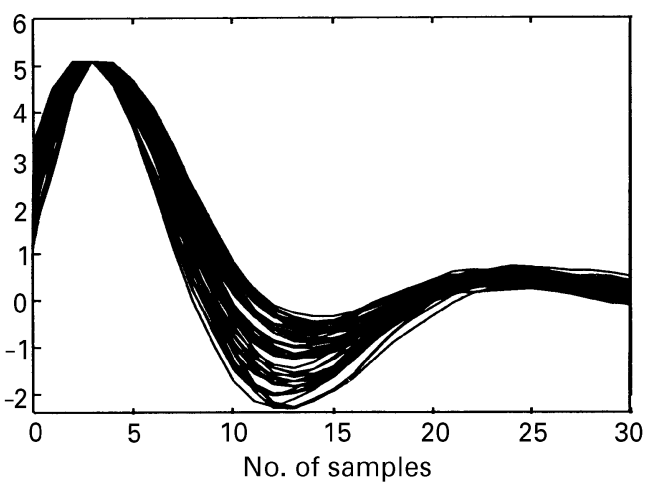

(a)

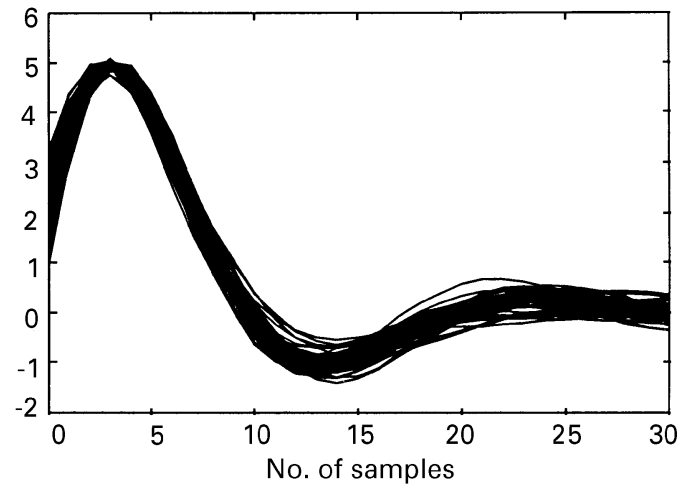

(b)

Figure 2. Channel identification for $H_{1}(z)$. (a) Existing method, (b) proposed method. 
along the frequency $(f)$ axis. The SCD is estimated at $\alpha=0$ for magnitude estimation and at $\alpha=N / p$ (corresponding to $l=1$ in (12)) for phase estimation..

The MGD is calculated by considering the first $M_{1}$ cepstral coefficients. $M_{1}=30$ and 10 for $H_{1}(z)$ and $H_{2}(z)$ respectively. The smoothed PGD, after correction using MGD, is also estimated by considering the first $M_{2}$ cepstral coefficients. $M_{2}=30$ and 35 for $H_{1}(z)$ and $\mathrm{H}_{2}(z)$ respectively.

For $H_{1}(z)$, figures $2 \mathrm{a}$ and $\mathrm{b}$ indicate that the mean estimate obtained by the proposed method follows the true impulse response better than the original method. The RMSE plot indicates that the variance of the estimate is significantly improved by the proposed method. Also, the individual estimates (shown in figure 2) support this result.

The \%NMSE without any phase correction is 6.83 and that with phase correction by GD approach is 3.50 . Thus, there is an improvement in performance of about $50 \%$, by the proposed GD approach over the existing one.

The results for $H_{2}(z)$ are given in figure 3. The results are similar to those for $H_{1}(z)$. The $\%$ NMSE without any correction for phase is 11.55 and that for phase correction by the GD approach is 7.86 . Thus, there is an improvement in performance of about $32 \%$, by the GD approach.

The performance of proposed channel identification for the above systems under noisy conditions is considered. The additive noise is white Gaussian and the SNR is $12 \mathrm{~dB}$. The oversampling rate $p$ is taken to be 3 , an odd number, in order to comply with the constraint presented in $\S 2$, for magnitude identification, under noisy condition.

A data length $N=8,192$ is used. The SCD smoothed over 201 products along the frequency $(f)$ axis is estimated at $\alpha=N / p$ (corresponding to $l=1$ in (12)) both for
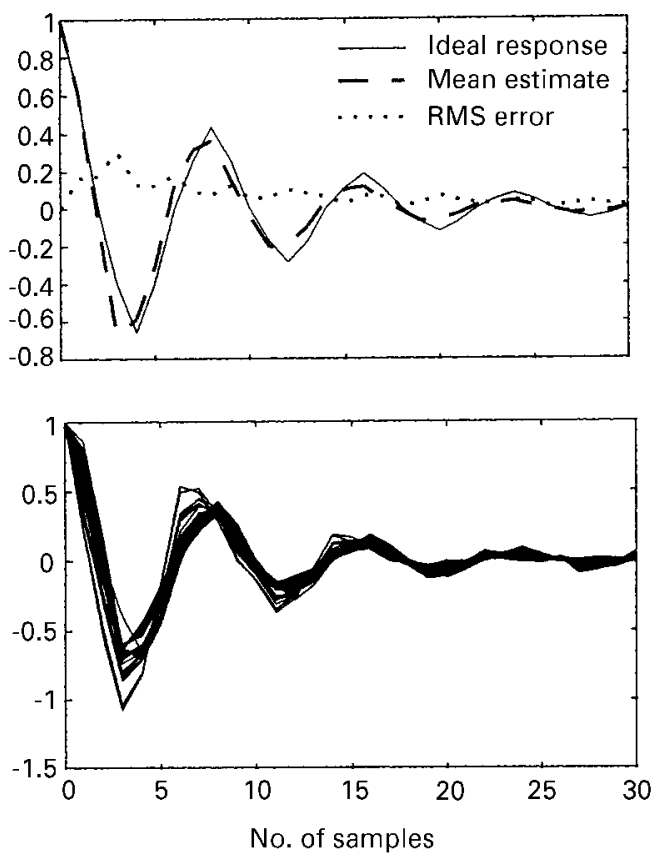

(a)
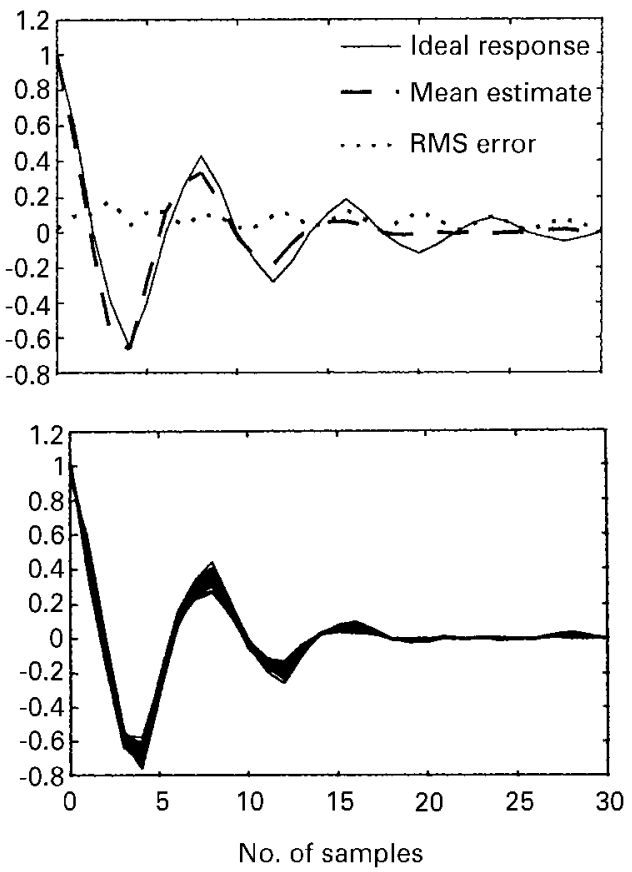

(b)

Figure 3. Channel identification for $H_{2}(z)$. (a) Existing method, (b) proposed method. 


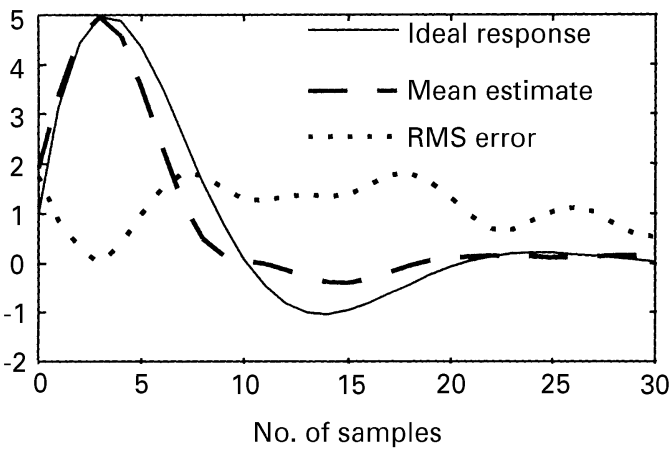

(a)

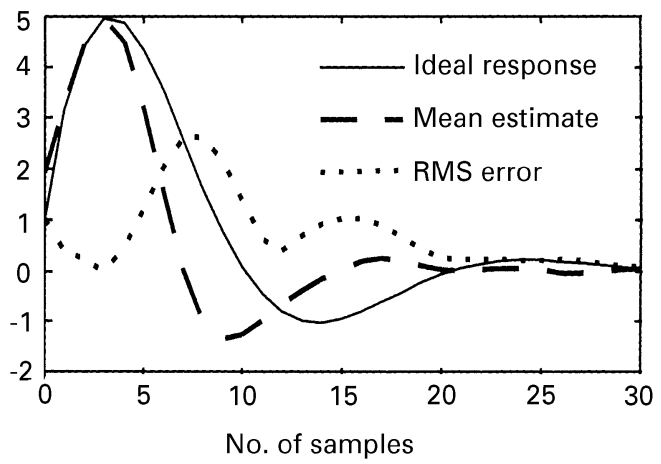

(b)

Figure 4. Channel identification for $H_{1}(z)$ with an SNR of 12dB. (a) Existing method, (b) proposed method.

magnitude and phase estimation. The values of $M_{1}$ and $M_{2}$ used are same as for the clean signal. The results are shown in figures 4 and 5 .

For $H_{1}(z)$, the \%NMSE without any phase correction is 41.23 and that by the GD approach is 27.12 . Thus, the improvement provided by the GD approach, in this case is only $34 \%$, as compared to $49 \%$ under noiseless condition.

For $H_{2}(z)$, the $\%$ NMSE without any phase correction is 75.10 and that by the GD approach is 60.10 . Thus, the improvement provided by the GD approach, in this case is only $20 \%$, as compared to $32 \%$ under noiseless condition.

From the results it is evident that the proposed phase correction based on GD provides a significant improvement in the channel estimate in terms RMSE over the existing method, both under noiseless and noisy conditions.x

\section{Conclusions}

A new method based on second order cyclostationary statistics and properties of group delay, was considered for blind identification of a system. This was achieved by applying a

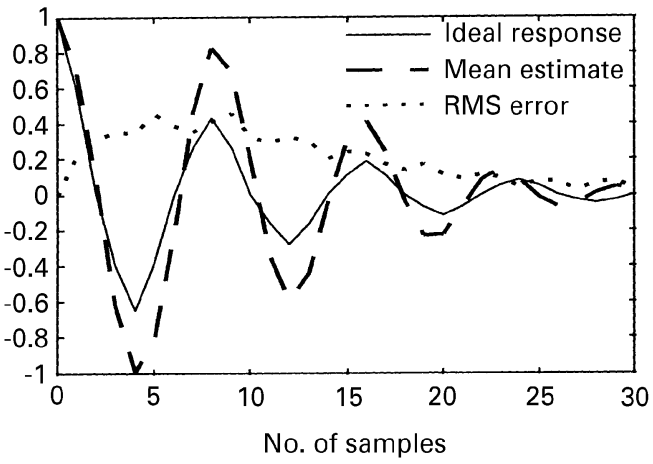

(a)

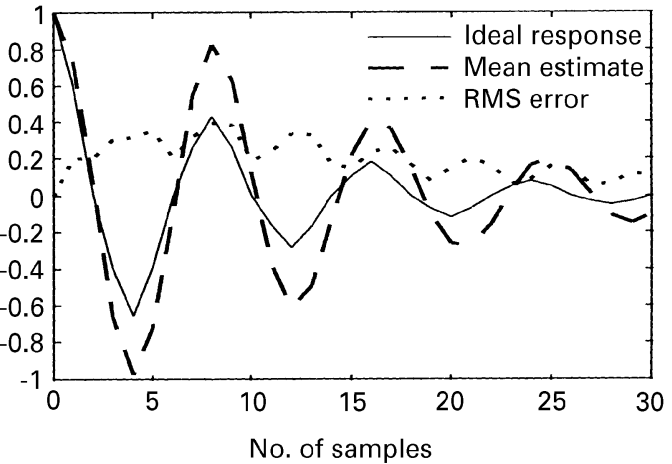

(b)

Figure 5. Channel identification for $H_{2}(z)$ with an SNR of $12 \mathrm{~dB}$. (a) Existing method, (b) proposed method. 
correction to the estimated phase (by the spectral correlation density of the system output) for the poles, in the group delay domain. The results indicated a significant improvement in the blind identification, in terms of root mean square error. Depending upon the SNR and the system under consideration, the improvement in percentage normalized mean square error was found to be in the range of 20 to $50 \%$. The GD domain not only facilitates easy phase correction, but being a nonparametric method, also provides better performance at low signal-to-noise ratios and is applicable to signals in general.

\section{References}

Gardner W A 1991 Exploitation of spectral redundancy in cyclostationary signals. IEEE Signal Process. Mag. 8: 14-36

Giannakis G B, Mendel J M 1988 Identification of nonminimum phase systems using higher order statistics. IEEE Trans. Acoust., Speech, Signal Process. 37: 360-377

Hatzinakos D 1994 Nonminimum phase channel deconvolution using the complex cepstrum of the cyclic autocorrelation IEEE Trans. Signal Process. 42: 3026-3042

Li Y, Ding Z 1994 ARMA system identification based on second-order cyclostationarity. IEEE Trans. Signal Process. 42: 3483-3494

Narasimhan S V, Reddy G R, Plotkin E I, Swamy M N S 1992a Phase estimation by bispectrum: A group delay approach. Signal Process. 27: 79-86

Narasimhan S V, Reddy G R, Plotkin E I, Swamy M N S 1992b Bispectrum based mixed phase system identification by AR/ARMA models: A group delay approach. IEEE Trans. Circuits Syst. II 39: 671-674

Nikias C L 1988 ARMA bispectrum approach to nonminimum phase system identification. IEEE Trans. Acoust., Speech, Signal Process. 36: 513-525

Tsatsanis M K 1997 Transmitter induced cyclostationarity for blind channel equalization. IEEE Trans. Signal Process. 45: 1785-1794

Yegnanarayana B 1981 Speech analysis by pole-zero decomposition of short time spectra. Signal Process. 3: 5-17

Yegnanarayana B, Saikia D K, Krishnan T R 1984 Significance of group delay functions in signal reconstruction from spectral magnitude or phase. IEEE Trans. Acoust., Speech Signal Process. ASSP-32: 610-623 